\title{
El Bienestar Infantil: su medición y dimensiones relevantes para los primeros años de vida ${ }^{1}$
}

Victoria Tenenbaum ${ }^{2}$

Fedora Carbajal ${ }^{3}$

Instituto de Economía - Universidad de la República del Uruguay

\section{Artículo científico}

Material original autorizado para su primera publicación en el Journal de Ciencias Sociales, Revista Académica de la Facultad de Ciencias Sociales de la Universidad de Palermo.

Recepción: 29-11-2019

Aceptación: 29-07-2020

Resumen: El bienestar en primera infancia es relevante principalmente en los países en desarrollo y se considera pertinente el hecho de generar evidencia que ayude a comprender y repensar las políticas con foco en esta etapa de la vida. El objetivo de este estudio es analizar las principales dimensiones vinculadas al bienestar infantil, en particular el desarrollo infantil (ASQ 3 y CBCL), el estado nutricional, la crianza, la situación de la vivienda y la protección social vinculada a la situación educativa y laboral de las madres. Mediante estas dimensiones se construye un índice de bienestar infantil (IBI) con la metodología de Alkire y Foster, y se exploran algunos de los canales que afectan al índice como puede ser la asistencia educativa temprana y la salud mental de la madre para el caso uruguayo. El índice de bienestar infantil arroja un resultado de 0.892 , mostrando que alrededor de $34 \%$ de los niños entre 2 y 6 años de edad en Uruguay presentan ausencia de bienestar siendo las dimensiones de Desarrollo Infantil y Protección social las que mayormente contribuyen. El 66\% de los niños restantes cuentan con un nivel suficiente de bienestar, mostrando que cuentan con un nivel satisfactorio de bienestar en un $68 \%$ de los indicadores en promedio. Adicionalmente, se estudian las diferencias en términos de suficiencia y ausencia de bienestar según edad, región, asistencia

\footnotetext{
${ }^{1}$ Este estudio fue realizado gracias al financiamiento del Fondo concursable Iniciación a la investigación de CSIC, UdelaR - Uruguay. Las autoras agradecer, particularmente, la coordinación de Gonzalo Salas.

${ }^{2}$ Economista- Facultad de Ciencias Económicas y Administración, Universidad de la República de Uruguay. Master en Economía Aplicada de la Universidad Autónoma de Barcelona. Consultora de la oficina de CEPAL en Montevideo. Docente e Investigadora del Instit. de Economía de la Facultad de Ciencias Económicas y Administración, UdelaR Uruguay. Correo electrónico: victenen@gmail.com

${ }^{3}$ Economista- Facultad de Ciencias Económicas y Administración, Universidad de la República de Uruguay. Master en Economía de la Universidad Nacional de La Plata, Argentina.Consultora del Centro de Investigaciones Económicas (cinve) y de la Oxford Poverty and Human Development Initiative (OPHI), Universidad de Oxford, Reino Unido. Docente e Investigadora del Instituto de Economía de la Facultad de Ciencias Económicas y Administración, UdelaR Uruguay. Correo electrónico: fedorace@gmail.com
} 
educativa, sexo, en este último caso se encuentra que los varones cuentan con menor nivel de bienestar relativo.

Palabras Clave: bienestar infantil; pobreza multidimensional; primera infancia, desarrollo socioemocional.

\section{Child Well-being: its measurement and relevant dimensions}

Abstract: Promoting the development of children's social well-being in early childhood is a huge challenge for developing countries. Studying the relationship between the main components of early childhood well-being helps to understand and rethink policies focused on this stage of life. The aim of this study is to analyze the main dimensions related to children well-being, particularly child development (ASQ 3 and CBCL), nutritional status, parenting practices, household characteristics and mother's social protection. Through these dimensions, an index of child welfare (IBI) was constructed with Alkire y Foster methodology using a novel database, and some of the channels that affect the index were explored, such as the early educational attendance and mental health of the mother. The child well-being index for Uruguay shows a result of 0.892 , implying that around $34 \%$ of children between 2 and 6 years old are deprived on well-being, being the dimensions of Child Development and Social Protection the ones that contribute the most. $66 \%$ of the remaining children have a sufficient level of well-being, showing that they have on average sufficient well-being in $68 \%$ of the indicators. Additionally, differences in terms of sufficiency and absence of well-being were studied according to age, region and sex, being the last characteristic where greater differences are verified, showing lower well-being in boys.

Keywords: child well-being; multidimensional poverty; early childhood; socio emotional development.

\section{Introducción}

Numerosos estudios remarcan la importancia de la primera infancia como etapa fundamental en el desarrollo físico y mental de las personas, a la vez que muchas de las deficiencias que provienen de esa etapa implican reducciones en el bienestar futuro que pueden significar problemas complejos para la sociedad. Existe evidencia que programas implementados en etapas posteriores del desarrollo de un individuo son menos efectivos y tienen menores retornos a nivel individual que aquellos orientados a la primera infancia (Biggeri, 2004; Conti y Heckman, 2012; Cunha en al., 2006).

En este sentido, el enfoque de las capacidades de Sen (1992) proporciona un marco teórico para la medición del bienestar en los niños ya que existen capacidades específicas en esta fase de la vida. Asimismo, la capacidad de los niños de convertir recursos y bienes 
en capacidades y funcionamientos depende de factores de conversión individual y social, en especial de las capacidades de sus padres o cuidadores. Las capacidades de los padres pueden afectar directa o indirectamente a las del niño, existiendo una transferencia intergeneracional de estas capacidades. Sen menciona que las capacidades se pueden adquirir de forma dinámica, pues las desarrolladas tanto en la infancia como en la adolescencia son decisivas para la vida, afectando capacidades y logros en la vida adulta.

Las capacidades también se ven afectadas por los funcionamientos de sus padres, como es el vínculo entre la salud materna y la nutrición del niño o entre la educación de los padres y los niños. Esto puede entrar en conflicto con los deseos o libertades del niño, ya que este no es un actor pasivo. Su grado de autonomía varía según las edades, en particular en lo referido a determinadas capacidades para expresar sus ideas. Bajo esa concepción se ubica al niño como centro de transferencia intergeneracional de las capacidades, pero a su vez, como motor de cambio de estas (Biggeri, 2004). De esta manera, es necesario comprender de forma precisa cuáles son las dimensiones determinantes del bienestar en la primera infancia.

En Uruguay la pobreza monetaria se redujo significativamente en los últimos años, sin embargo, continúa siendo un problema de relevancia para el país que se acentúa en etapas tempranas de la vida. Esto tiene un correlato con países de América Latina donde si bien hubo logros en los últimos 15 años en disminución de la pobreza, el logro de la suficiencia en el bienestar infantil continúa siendo un problema relevante.

Este estudio se propone construir un indicador de bienestar infantil que permita caracterizar de forma lo más abarcativa posible la situación de la primera infancia, definiendo las dimensiones que mejor se ajustan y reflejan el bienestar multidimensional, e intenta conocer algunos de los canales por los cuales se manifiestan. Así, en primer lugar, se desarrolla un indicador de bienestar con algunas dimensiones que no han sido exploradas con anterioridad al contar con una nueva fuente de información, única para el análisis de la primera infancia en el país. En segundo lugar, es un tema muy relevante en países en desarrollo ya que, si bien en los últimos años han existido mejoras sustanciales en la pobreza, los antecedentes muestran que siguen existiendo importantes privaciones para este grupo etario que no se han podido superar. En tercer lugar, porque estudiar el bienestar en la infancia y los canales que vinculan sus dimensiones con determinadas políticas es relevante a la hora de pensar políticas adecuadas para esta etapa del desarrollo.

Se propone un Índice de Bienestar Infantil (IBI) utilizando la metodología de Alkire y Foster (2011) en base a la Encuesta de Nutrición, Desarrollo Infantil y Salud (ENDIS). Se incorporan dos tests de desarrollo infantil frecuentemente utilizados en la literatura especializada (ASQ3 y CBCL); prácticas de crianza y apoyo en la crianza aspectos relativos 
a nutrición y salud; se incluyen indicadores relacionados a la vivienda, y por último una dimensión vinculada a la situación laboral y educativa de la madre. Se analiza la asociación entre este indicador y posibles canales que afectan el bienestar que han sido estudiados en la literatura, como la asistencia educativa, la salud mental y otros rasgos de personalidad y entorno familiar de la madre.

\section{Antecedentes y estado del arte}

Es habitual que el enfoque multidimensional utilice como marco normativo el enfoque de las capacidades de Amartya Sen (1992), a partir de que se pueden adoptar distintas decisiones metodológicas para la medición del bienestar (Biggeri et al. 2011). La pobreza para Sen (1992) es definida como el fracaso para obtener un mínimo de capacidades básicas para la vida, no permitiendo esas privaciones el logro de otras capacidades. Si bien los recursos como el ingreso $\mathrm{u}$ otros activos son importantes para generar las capacidades y funcionamientos, cumplen un claro rol instrumental (Robeyns, 2003). Los funcionamientos son aquellos logros por los cuales las personas consiguen ser o hacer, como tener un buen estado de salud o una buena educación. Sen $(1992,1993)$ plantea que la libertad de cada individuo se refleja en las diferentes combinaciones de esos funcionamientos dentro de los cuales cada persona pueda elegir.

En la literatura académica reciente se han llevado a cabo diversos esfuerzos para intentar medir el bienestar infantil, en particular en primera infancia, con diferentes criterios y grados de alcance (Andresen et al. 2010; Biggeri et al. 2010). En general, se entiende que el desarrollo infantil comprende cuatro dimensiones que abarcan el dominio físico o motor, el cognitivo, el emocional y el social (UNICEF, 2012).

En particular, los estudios sobre pobreza multidimensional orientados a la infancia relevan información relativa a estas edades que expresan las dimensiones de mayor relevancia (Biggeri et al. 2011; Alkire et al. 2016). El enfoque de Bristol significó un primer acercamiento a proveer una metodología de medición multidimensional de la pobreza infantil, generando la primera medida comparable entre países en desarrollo mediante un enfoque que se basa en los derechos del niño (Roelen et al. 2012; Stefánsson et al. 2018). Existen otros estudios de referencia que también utilizan dimensiones propias de los niños, construyendo índices mediante la metodología de Alkire y Foster (2011) que ha sido extensamente aplicada en países en desarrollo por parte de Oxford Poverty and Human Development Initiative (OPHI) (Alkire et al. 2016).

Numerosos trabajos se centran en el estudio de las dimensiones del bienestar infantil y los canales que pueden afectarlas. Por un lado, aquellos que estudian los impactos de 
distintos programas específicos, como puede ser la asistencia preescolar sobre el desarrollo infantil. Aunque los resultados difieren entre estudios, en general la mayoría indica efectos positivos sobre el desarrollo de los niños y niñas, obteniendo mayores efectos para hogares de menores ingresos y/o en centros de mayor calidad (Blau y Currie, 2006; Ahnert y Lamb, 2003), en madres menos educadas (Felfe y Lalive, 2014), o en edades específicas (Urzúa y Varamendi, 2011). Adicionalmente, otro grupo de estudios analizan los determinantes o canales del desarrollo infantil como pueden ser las prácticas de crianza y el ambiente familiar. Si bien no existe un consenso sobre la forma en que debe ser la crianza parental para generar mejores resultados, algunos estudios muestran que una crianza afectiva y estimulante impacta en el desarrollo cerebral del niño a través de un mayor desarrollo de las conexiones y patrones neuronales (Cunha et al, 2006). Conti y Heckman (2012) resumen varios canales que influyen en el bienestar infantil. Los autores señalan que la inversión realizada por los padres, la salud del niño, los antecedentes familiares, la transmisión intergeneracional de habilidades y las condiciones de vida en la primera infancia son los principales determinantes (Cunha et al. 2006; Almond y Currie, 2011).

Para Uruguay existen algunos estudios sobre pobreza multidimensional o bienestar infantil que mediante el enfoque de las capacidades identifican dimensiones para niños y adolescentes (Nathan y Zerpa, 2011; Alves y Zerpa, 2011; Castillo y Colombo, 2014; Failache et al. 2016; Colacce y Tenenbaum, 2017).

Son tres los antecedentes nacionales más relevantes para nuestro estudio los cuales construyen indicadores de primera infancia con encuestas específicas. Por un lado, utilizando la ENDIS, Pérez (2016) construye un índice de pobreza multidimensional para estudiar los determinantes de la pobreza infantil. Colacce y Tenenbaum (2018) plantean en base a las dos olas de la ENDIS un índice multidimensional en primera infancia para el análisis de la focalización del programa Uruguay Crece Contigo (UCC) y utilizan las dimensiones de Características del Hogar (vivienda y educación de la madre), Salud del niño, Embarazo, Nutrición (seguridad alimentaria) y Prácticas de Crianza. Por otro lado, Marroig et al. (2017) para la evaluación del programa UCC, proponen un índice de pobreza multidimensional que comprende indicadores sanitarios, talla y peso para la edad, semanas de gestación, peso al nacer, VDRL en embarazo y número de controles.

Si bien los antecedentes constituyen referencias importantes y delimitan -en partelas dimensiones relevantes a considerarse en Uruguay, cuentan con una serie de limitaciones que la presente propuesta de investigación intenta abordar. En primer lugar, los trabajos precedentes no incorporan indicadores de desarrollo infantil en la primera infancia, que como se mencionó en el diagnóstico sobre el desarrollo en esta etapa de la vida de los individuos resulta clave tanto para su desempeño futuro a nivel individual como respecto a los derrames 
que genera a la sociedad en su conjunto. En segundo lugar, son menos los estudios que utilizan bases de datos específicas para la población infantil lo cual reduce la capacidad de las medidas de pobreza multidimensional a ajustarse a características particulares de dicho tramo etario. En tercer lugar, ninguno de esos estudios explora algunos de los canales principales por los cuáles puede verse afectado un índice de bienestar multidimensional en primera infancia, enfocado en el desarrollo infantil.

\section{Metodología y fuente de información}

\subsection{Fuente de Información}

Se utiliza como fuente de datos la Encuesta de Nutrición, Desarrollo Infantil y Salud (ENDIS) elaborada por el Instituto Nacional de Estadística (INE) en conjunto con el Grupo de Estudios de Familia de la Universidad de la República y el Programa Uruguay Crece Contigo. ${ }^{4}$ El universo de la encuesta son hogares con niños de 6 a 48 meses en 2013/14, que fueron entrevistados previamente por la Encuesta Continua de Hogares $(E C H)$. La encuesta cuenta con la característica de ser una base de datos en panel y se consideran las dos olas de relevamiento (2013-14 y 2015-16). ${ }^{5}$ La primera ola cubrió 2,665 hogares particulares donde residían 3,077 niños para localidades urbanas mayores a 5,000 habitantes. En la segunda ola se entrevistó a 2,085 hogares de los presentes en la primera ola, incluyendo 2,383 niños. En este estudio se utiliza la segunda ola de la ENDIS que tiene 2,283 observaciones para niños/as entre 2 y 6 años residentes en hogares particulares (sin hogares secundarios). ${ }^{6}$

La ENDIS provee información comprehensiva para la primera infancia en Uruguay y tiene la particularidad de haber sido específicamente diseñada para el estudio de la población en este tramo etario. Recaba información sobre características socioeconómicas, de vivienda y demográficas de los hogares, el estado nutricional de los niños a través de mediciones antropométricas y prácticas de alimentación, desarrollo infantil, prácticas de crianza, cuidados, condiciones de salud de la mujer y del transcurso del embarazo, así como sobre el acceso a los servicios de salud y educación preescolar.

\footnotetext{
${ }^{4}$ En Chile y Colombia existe un correlato con la ENDIS ya que ambos cuentan con encuestas sobre desarrollo infantil longitudinales con al menos dos rondas de recolección (ELPI y ELCA, respectivamente) en los que se relevan entre otras las dimensiones de nutrición y desarrollo socioemocional. En los tres países las diferencias son relevantes en términos de condición socioeconómica del hogar a la que pertenece el niño/a.

${ }^{5}$ Por características específicas de la encuesta y sobre sus resultados para la primera ola véase Grupo de Estudios de Familia (2015).

${ }^{6}$ Una de las razones por las cuales se utiliza la segunda ola es que las pruebas psicométricas en la primera ola sólo fueron aplicadas en la capital del país, mientras que en la segunda se realizaron a nivel nacional.
} 


\subsection{Indicador de Pobreza Multidimensional}

La visión de Sen del enfoque de capacidades y funcionamientos puede ser operativizada a través de la construcción de una medición de pobreza multidimensional. Cualquier construcción de un indicador que intente medir la pobreza involucra así tres pasos: a) la elección del espacio; b) la identificación de quien es considerado en situación de pobreza y c) la agregación. El paso fundamental consiste en identificar quien es considerado pobre de acuerdo con un conjunto de dimensiones. Si bien en cada paso y etapa de construcción del indicador de pobreza se establecen necesariamente juicios normativos, los indicadores multidimensionales de pobreza han sido empleados ampliamente en la literatura empírica motivados por diferentes enfoques conceptuales.

Una aproximación metodológica que ha ganado creciente interés es la familia de medidas de pobreza multidimensional de Alkire y Foster (2011). El indicador de Alkire y Foster emplea una extensión multidimensional $\left(M_{\propto}\right)$ a la familia de medidas unidimensional conocidas como FGT. El indicador $M_{\propto}$ posee una serie de ventajas que cabe destacar. En primer lugar, satisface un conjunto de propiedades deseables de un indicador de pobreza, entre las que se subrayan, las de monotonicidad y descomposición. En segundo lugar, es un método de línea de "corte dual" y esto lo torna flexible. Se establecen umbrales para cada indicador y para cada dimensión y una vez agregado se fija un punto de corte que refleja cuántas dimensiones (o en qué proporción) son las necesarias para considerar a un individuo en situación de pobreza. ${ }^{7}$ En tercer lugar, este indicador permite la elección de la ponderación a utilizar para cada dimensión en la medición agregada de pobreza, y de cada indicador en cada una de las dimensiones, permitiendo la discrecionalidad de la elección.

La etapa de agregación consiste en calcular la proporción de individuos identificados como multidimensionalmente pobres (la incidencia multidimensional de la pobreza, $\boldsymbol{H}$ ) y la participación promedio de los indicadores ponderados para los cuales los individuos multidimensionalmente pobres están privados (definida como la intensidad de la pobreza multidimensional, $\boldsymbol{A})$. De esta manera, es posible construir el indicador de pobreza multidimensional de la forma:

$$
M_{0}=H \times A
$$

Como fue planteado el indicador $M_{0}$ expresa una medida de insuficiencia o privación multidimensional. Sin embargo, Ura, Alkire y Zangmo (2012) plantean para el caso de Bhutan

\footnotetext{
${ }^{7}$ Esto lo hace diferente de otros indicadores de pobreza multidimensional que persiguen el criterio de unión (un individuo está en situación de pobreza si está privado en al menos una dimensión) o de intersección (un individuo es pobre multidimensional si está privado en todas las dimensiones). Dichas alternativas extremas son contempladas en el indicador de Alkire y Foster (2011), así como situaciones intermedias.
} 
un indicador que denominan indicador generalizado de felicidad (IGF) que refleja ausencia de privaciones, en este caso se denomina Índice de Bienestar Infantil (IBI). En el presente estudio. siguiendo a Ura et al. (2012). se considerará este último indicador planteado con el propósito de dar cuenta del nivel suficiente de bienestar de los niños y niñas. Esto significa que se analizarán las dimensiones del bienestar para los niños y niñas que se encuentran por encima del umbral determinado.

Siguiendo a Main y Bradshaw (2012), bienestar y privación refieren a un mismo elemento en sentidos contrapuestos, mientras la pobreza multidimensional refiere a la privación en determinados derechos o dimensiones, el bienestar se vincula con la satisfacción de derechos y dimensiones que se incluyen en un indicador. Así, el IBI se expresa como 1 $M_{0}=1-H^{*} A$, dando cuenta de una proporción de la población que presenta suficiencia 0 bienestar multidimensional, dadas las decisiones consideradas para el doble corte.

En ese entendido, $I B I=1-M_{0}$ expresa el conteo $H$ de veces que en promedio las dimensiones en las que los niños alcanzan el nivel de suficiencia de bienestar (1- $A$ ). Realizando algunas operaciones aritméticas este indicador se puede expresar como (Ura et al. 2012): $I B I=H b+(H i * A b)$ donde $A b=1-A i$, y donde $H b$ es la proporción de niños que tienen suficiencia en el bienestar sumado al producto de $(H i * A b)$ que representa la proporción de aquellos que tienen bienestar insuficiente por el porcentaje de dimensiones en las cuales en promedio no tienen bienestar suficiente. Esta última forma es de utilidad para explicar de manera intuitiva el término, aunque es indistinto verlo de una u otra forma. Es importante aclarar que el IBI también permite descomponerse por subgrupos e indicadores cumpliendo las mismas propiedades que el índice de pobreza multidimensional $M_{0}$.

\subsubsection{Selección de dimensiones e indicadores}

Sen sugiere que cada sociedad debería definir su listado de funcionamientos y capacidades necesarias, que no pueden determinarse de forma universal. Esto es un punto de controversia reflejando la complejidad que tiene la operacionalización de este enfoque: el poder convertir las capacidades en indicadores plausibles de medir (Minujin et al. 2006). En ese sentido, Nussbaum (2000) plantea la necesidad de definir un conjunto de capacidades y elabora un listado de capacidades básicas el que es retomado en numerosos estudios.

Los antecedentes ya mencionados realizan distintas aproximaciones para la selección de las dimensiones que van desde la disponibilidad de información en una única fuente de datos, aspectos normativos como el listado de Nussbaum (2000), los consensos públicos como lo es la Declaración Universal de los Derechos Humanos, e incluso se consideran procesos participativos en los que se consulta a los actores como es el caso del estudio de 
Biggeri et al. (2006) para infancia y adolescencia. Son varios los estudios a nivel internacional que definen dimensiones relevantes de la infancia para la medición de la pobreza multidimensional y dependiendo de la disponibilidad de información se enfocan en dimensiones específicas de los niños además de dimensiones que dan cuenta de características de sus hogares (Alkire et al. 2016; Trani y Cannings, 2013).

Por su parte, Biggeri et al. (2011) mediante la consulta a niños y niñas en base a un listado de capacidades, plantean cuáles son las más relevantes, se constatan niveles de importancia distintos según la edad y grado de autonomía del niño. Entre las principales enumeradas por los niños aparecen amor y cuidado, vida y salud física, ocio y educación (por su valor presente y como instrumento para el futuro). En el caso de otras capacidades como el tiempo libre y autónomo y la movilidad se constata un aumento de su relevancia con la edad del niño, y otras van en dirección opuesta a la edad como amor y cuidado que es de las más relevantes en los primeros años de vida y disminuye un poco conforme aumenta la edad.

En general, los estudios sobre infancia que tienen un enfoque multidimensional coinciden en la mayoría de las dimensiones utilizadas, aunque no en sus indicadores debido a que en gran medida dependen de la disponibilidad de información en cada país. En CEPALUNICEF (2010) estudian las privaciones multidimensionales de los niños en América Latina basándose en un indicador con las siguientes dimensiones: nutrición, agua potable, servicios sanitarios, salud, vivienda, educación e información. Posteriormente a dicho estudio se realizaron varios en los que el núcleo principal de dimensiones son las asociadas a las características del hogar, aunque en ocasiones se incluyen otras consideradas relevantes en cada realidad nacional como puede ser el trabajo infantil o las condiciones de cuidado infantil (Espíndola et al. 2017). En un estudio para Colombia, Torres (2014) considera la dimensión de maltrato infantil, y Trani y Cannings (2013) para el caso de Darfur Occidental incorporan algunas dimensiones poco frecuentes como participación social, cuidado y amor de los padres y bienestar psicológico. En el caso de Tuñón et al. (2015), trabajaron la dimensión de estimulación temprana en un estudio para Argentina en el que incorporaron como indicadores: leer cuentos, jugar con ellos, asistencia a centros educativos y maltrato físico y/o verbal.

En el presente trabajo, para la selección de indicadores se exploró la literatura existente incorporando algunas dimensiones mencionadas anteriormente y que en Biggeri et al. (2011) son enumeradas (aquellas que están disponibles en nuestra base de datos), y posteriormente varios estudios empíricos las toman en cuenta para indicadores en infancia como los antes expuestos (Alkire y Roche, 2011; Alkire et al. 2016; Trani y Cannings, 2013; Apablaza y Yanoletsky, 2011). En el siguiente cuadro se presentan las dimensiones e indicadores seleccionados, así como el punto de corte mediante el cual se considera la 
privación y el peso de cada una ${ }^{8}$. Se le otorga el mismo ponderador a todas las dimensiones $(20 \%)$ e igual peso a cada indicador al interior de cada dimensión.

Cuadro 1. Dimensiones e Indicadores del Índice de Bienestar Infantil (IBI)

\begin{tabular}{|c|c|c|c|}
\hline Dimensión & Indicador & Punto de corte & $\begin{array}{l}\text { Peso sin } \\
\text { clima (\%) }\end{array}$ \\
\hline \multirow[t]{2}{*}{ Desarrollo infantil } & ASQ3 & $\begin{array}{l}\text { Nivel de riesgo o monitoreo en al menos una de las áreas: } \\
\text { comunicación, motricidad gruesa, motricidad fina, resolución } \\
\text { de problemas, personal social }\end{array}$ & $1 / 10$ \\
\hline & CBCL & Nivel de límite o clínico & $1 / 10$ \\
\hline \multirow{3}{*}{ Crianza y redes } & Autoritario & $\begin{array}{l}\text { Presenta más de un desvío estándar en el indicador de } \\
\text { autoritario }\end{array}$ & $1 / 15$ \\
\hline & Juego & $\begin{array}{l}\text { No acostumbra a enseñarle juegos, o cantarle canciones, o } \\
\text { contarle cuentos o inventarles historias }\end{array}$ & $1 / 15$ \\
\hline & Redes y Apoyo & $\begin{array}{l}\text { No tiene (o rara vez tiene) contacto o le ayudan a criar a su } \\
\text { hijo con padres, suegros o o padres de su ex pareja, otros } \\
\text { familiares, amigos o vecinos; o cree que necesita apoyo o } \\
\text { sostén seguido o muy seguido y no tiene a quien recurrir }\end{array}$ & $1 / 15$ \\
\hline \multirow[b]{3}{*}{ Nutrición y Salud } & Talla & $\begin{array}{l}\text { Tiene menos de dos desvíos estándar en el indicador de } \\
\text { retraso de talla para la edad }\end{array}$ & $1 / 15$ \\
\hline & Obesidad & Alcanza el valor de 3 o más en el índice de masa corporal & $1 / 15$ \\
\hline & Controles y enfermedad & $\begin{array}{l}\text { Tuvo tos y fiebre, diarrea y fiebre, dolor de oidos, } \\
\text { respiración difícil o labios azules o morados y no se atendió; } \\
\text { y si excede el doble de tiempo en realizar el último control } \\
\text { pediátrico de acuerdo a la frecuencia de controles sugerida } \\
\text { por el Ministerio de Salud Pública }\end{array}$ & $1 / 15$ \\
\hline \multirow{3}{*}{ Vivienda } & Materiales de vivienda & Si los pisos, techos y paredes son modestos o precarios & $1 / 15$ \\
\hline & Hacinamiento & Más de 3 personas por habitación & $1 / 15$ \\
\hline & Servicios básicos & $\begin{array}{l}\text { El hogar no cuenta con calefón ni calentador de agua o } \\
\text { refrigerador o no cuenta con internet, tv o radio. }\end{array}$ & $1 / 15$ \\
\hline \multirow[b]{2}{*}{ Protección } & Educación de la madre & Máximo nivel de educación es primaria completa & $1 / 10$ \\
\hline & Proteccion social & $\begin{array}{l}\text { Si la madre está en situación de informalidad y no recibe } \\
\text { Asignaciones Familiares }\end{array}$ & $1 / 10$ \\
\hline
\end{tabular}

Fuente: elaboración propia en base a ENDIS.

En algunos estudios se combinan indicadores de asistencia educativa preescolar a partir de los 4 o 5 años, con indicadores vinculados al juego, la interacción con los adultos, estimulación oportuna en los primeros años de vida. Debido a que el estudio se enfoca en población entre 2 y 6 años no se incluyen indicadores de asistencia preescolar, aunque sí las otras dimensiones asociadas.

Para la medición del desarrollo infantil se utilizan pruebas psicométricas que se relevan en la segunda ola para todo el país, ASQ:3 y CBCL. Ambos son auto-administrados

\footnotetext{
${ }^{8}$ En el procedimiento de selección de los indicadores y umbrales se descartaron algunos de ellos debido a que contaban con insuficiente número de casos y sus tasas de incidencia eran reducidas, sus definiciones no resultaban operativas o resultaban redundantes en la estimación del índice. A modo de ejemplo, la identificación de si el niño contaba con anemia fue descartado por un número insuficiente de casos, en los indicadores sobre uso del tiempo en tareas de cuidado al niño no era posible identificar un umbral apropiado y objetivo, y se consideró la inclusión del indicador ASQ-SE, pero el aporte de la información que éste brindaba era similar al ASQ 3.
} 
por los padres o cuidadores y aplican a niños/as entre los 1 y 66 meses. El primero, ASQ:3, refleja el desarrollo psicomotriz y sus áreas son cinco: motricidad fina y gruesa, comunicación, resolución de problemas y socio personal. Los resultados se comparan con una tabla de puntos de corte según edad y se dividen en rangos normales, monitoreo y riesgo (Urzúa y Veramendi, 2011). En un estudio para Uruguay (Grupo de Estudios de Familia, 2015) se verificó que los niños de contexto de menor acceso a recursos tenían peores desempeños con excepción de la motricidad gruesa, en la que los bajos desempeños de los niños de quintiles altos equipararon a los de los más pobres. En este estudio se considera privado en este indicador si presenta nivel de riesgo o monitoreo en al menos una de las cinco áreas. El segundo indicador, $\mathrm{CBCL}$, identifica problemas socio-emocionales totales, se consideran por un lado problemas externalizados como lo es la falta de atención o conducta agresiva, e internalizados como la ansiedad, repliegue o aislamiento. Se calculan rangos para valores normal, límite y clínico (Urzúa y Veramendi, 2011). En este estudio se considera privado en este indicador aquellos niños que presentan rango límite o clínico.

La crianza es la configuración del espacio de interacción familiar, donde se relacionan aspectos vinculados a las habilidades, salud mental de los padres, aspectos culturales, características propias del niño, entre otros (Arranz, 2004). La dimensión identificada en este estudio es crianza y redes, la misma está integrada por tres indicadores.

En primer lugar, tipo de práctica de crianza autoritario refiere a la obediencia de los niños y el uso frecuente del castigo para regular su conducta. Se emplea como punto de corte que un niño es privado si las respuestas se encuentran a más de un desvío de la media (Perazzo et al. 2019). El segundo indicador que integra la dimensión denominada crianza y redes es el relativo a la interacción entre padres o adultos referentes del niño, y el niño (contarle cuentos, inventarle historias, cantarle o jugar habitualmente). Se considera privado en este indicador si responde que no acostumbra a realizar ninguna de esas actividades. Existen varios estudios que plantean estas actividades como fundamentales en los primeros años de vida ya que estas interacciones de la familia son los factores de mayor incidencia en el bienestar de los niños. A la vez, existen investigaciones neurológicas que encuentran que los cuidados cálidos y estimulantes al niño impactan en el desarrollo del cerebro por la vía del incremento de las conexiones y de los patrones neuronales (Berlinski y Schady, 2015). E tercero de los indicadores es el denominado redes y apoyos, se define a partir de dos variables relevadas en la ENDIS, no tener contacto alguno con familiares, vecinos o amigos que lo ayuden en la crianza, o no contar con apoyo o sostén alguno en caso de necesitarlo. Se determina privado en caso de que declaren no contar con nadie en ninguna de las dos.

La salud y nutrición se consideran aspectos relevantes del bienestar en sí mismos, y son condición necesaria para el acceso a otras funcionalidades, a la vez que en el caso de 
los niños pueden afectar la salud de las siguientes etapas del ciclo de vida y es necesario su prevención y control desde los primeros días. En este estudio se consideran tres indicadores.

El primero, mide resultados nutricionales a partir de talla para la edad el cual es asociado a condiciones adversas de la historia nutricional del niño, y se considera privación para aquellos que presenten retraso de talla con un valor inferior a 2 desvíos estándar del puntaje $z$ estandarizado calculado a partir de las tablas de la Organización Mundial de la Salud (OMS) ${ }^{9}$. El segundo indicador, obesidad y se define como el índice de masa corporal (IMC) mayor a tres desvíos. En este estudio se considera el índice de masa corporal debido a que en la segunda ola son niños de más de dos años y es el indicador propuesto para estas edades. El tercero, está vinculado a los controles de salud y la falta de atención en el caso de enfermedades graves, en particular se construye un indicador de controles de salud en función de la edad del niño y las metas planteadas por el Ministerio de Salud Pública. Se considera privado si no realiza un control pediátrico en más del doble de tiempo de lo estipulado por las metas y si declara no haberse atendido o consultado a un médico en caso de enfermedades que así lo requieren (diarrea y fiebre, tos y fiebre, respiración difícil, labios morados).

La cuarta dimensión considerada es vivienda, que busca brindar información de las condiciones materiales en las que vive el niño, en términos de su vivienda y del acceso a bienes básicos de confort. Esta dimensión se compone de tres indicadores. Por un lado, la privación en calidad de la vivienda mediante una categorización de materiales de piso, techo y paredes, donde se consideran privados aquellos que habitan las viviendas consideradas en la tipología como precarias y modestas (Colacce y Tenenbaum, 2018). Por otro lado, un indicador de hacinamiento en la vivienda (si hay tres personas o más por habitación destinada a dormir), y por último un indicador de tenencia de bienes de confort básicos como lo es que cuenten con calefón o calentador de agua y refrigerador o internet, televisión o radio.

La quinta dimensión denominada protección social se compone de dos indicadores, por un lado, el nivel educativo de la madre considerándose privado si su máximo nivel alcanzado es primaria o menos, lo que está asociado a menores posibilidades de empleo y a empleo formal. Adicionalmente, un indicador de privación laboral que incluye si la madre no cuenta con acceso a la seguridad social ni cuenta con asignaciones familiares ${ }^{10}$, lo cual es

\footnotetext{
${ }^{9}$ Estos valores se calculan utilizando el paquete de STATA igrowup elaborado por la OMS para los menores de 5 años y el paquete who2007 para los mayores de 5 años (segunda ola).

10 En su mayoría son de carácter no contributivo, se denominan Asignaciones Familiares del Plan de Equidad. Se crean en 2008 cubriendo alrededor de 400 mil menores de 18 años en situación de vulnerabilidad socioeconómica, lo que representa el $80 \%$ de los niños del primer quintil y el $50 \%$ de los del segundo quintil de ingresos per cápita a nivel de todo el país.
} 
una variable determinante en la reducción del riesgo de privaciones en la infancia (Pérez, 2016.

\subsubsection{Los canales asociados al bienestar infantil}

Se analizan algunos canales mediante los cuales se puede ver afectado el bienestar infantil, y que han sido explorados en la literatura como es el caso de la asistencia preescolar temprana, el bienestar psicológico de la madre mediante el test $S R Q$, y las características de personalidad de la madre mediante el Big Five Inventory (BFI) que en algunos de los rasgos se correlacionan con lo que mide el Self-Reporting Questionnaire (SRQ).

Como ya fue mencionado las experiencias de los primeros años de vida están moldeadas por la interacción del niño, su familia y cuidadores. Estas interacciones se producen en diferentes entornos como el hogar, el centro educativo inicial, la escuela, la comunidad, y a medida que los niños crecen va tomando más relevancia las instituciones educativas. Por ese motivo, la forma en que los niños asisten a un centro se vincula también con la calidad del mismo que también constituye un factor relevante en los resultados de desarrollo que presenta el niño (Berlinski y Schady, 2015).

Cabe resaltar que el tiempo y los recursos asignados por los individuos en los primeros años de vida, afectan desempeños posteriores y también presentes y en muchos casos presentan restricciones para los padres en sus decisiones sobre la asignación de su tiempo y recursos disponibles (Berlinski y Schady, 2015). El bienestar insuficiente puede también provocar una mayor incidencia de estrés y depresión en los cuidadores de un niño y en muchos casos en las madres, lo cual a su vez se ha asociado con peores resultados en el desarrollo infantil.

Por ese motivo, se incorporan instrumentos para la medición de personalidad, depresión y ansiedad. El BFI es una aproximación utilizada en muchos estudios y consensuada para medir personalidad, y se plantea que los rasgos de la personalidad son estables en la adultez. Se compone de cinco rasgos: neuroticismo, extraversión, apertura a la experiencia, responsabilildad y ser agradable y se utiliza el formulario que contiene 44 ítems. La segunda escala es el SRQ que se relaciona con uno de los rasgos de personalidad del BFI, es un instrumento de screening de los más comunes desórdenes mentales diseñado por la OMS (WHO,1994). En este caso se considera el SRQ 20 que contiene ítem vinculados al neuroticismo, en particular depresión, ansiedad y desórdenes de somatización.

\section{Resultados}




\title{
4.1. Incidencia de las dimensiones del bienestar
}

Se presentan las tasas de incidencia del bienestar para los niños/as entre 2 y 6 años de edad en los indicadores antes mencionados (Gráfico 1). El mayor bienestar relativo en este grupo de población es en la dimensión Nutrición y salud, principalmente en los indicadores de talla y obesidad y en redes y apoyo. En oposición, las tasas de incidencia más bajas están en ASQ3, Juego y Educación de la madre, entre alrededor de un $65 \%$ y $81 \%$ de los niños que cuentan con nivel aceptable de bienestar tienen suficiencia en estos indicadores. ${ }^{11}$

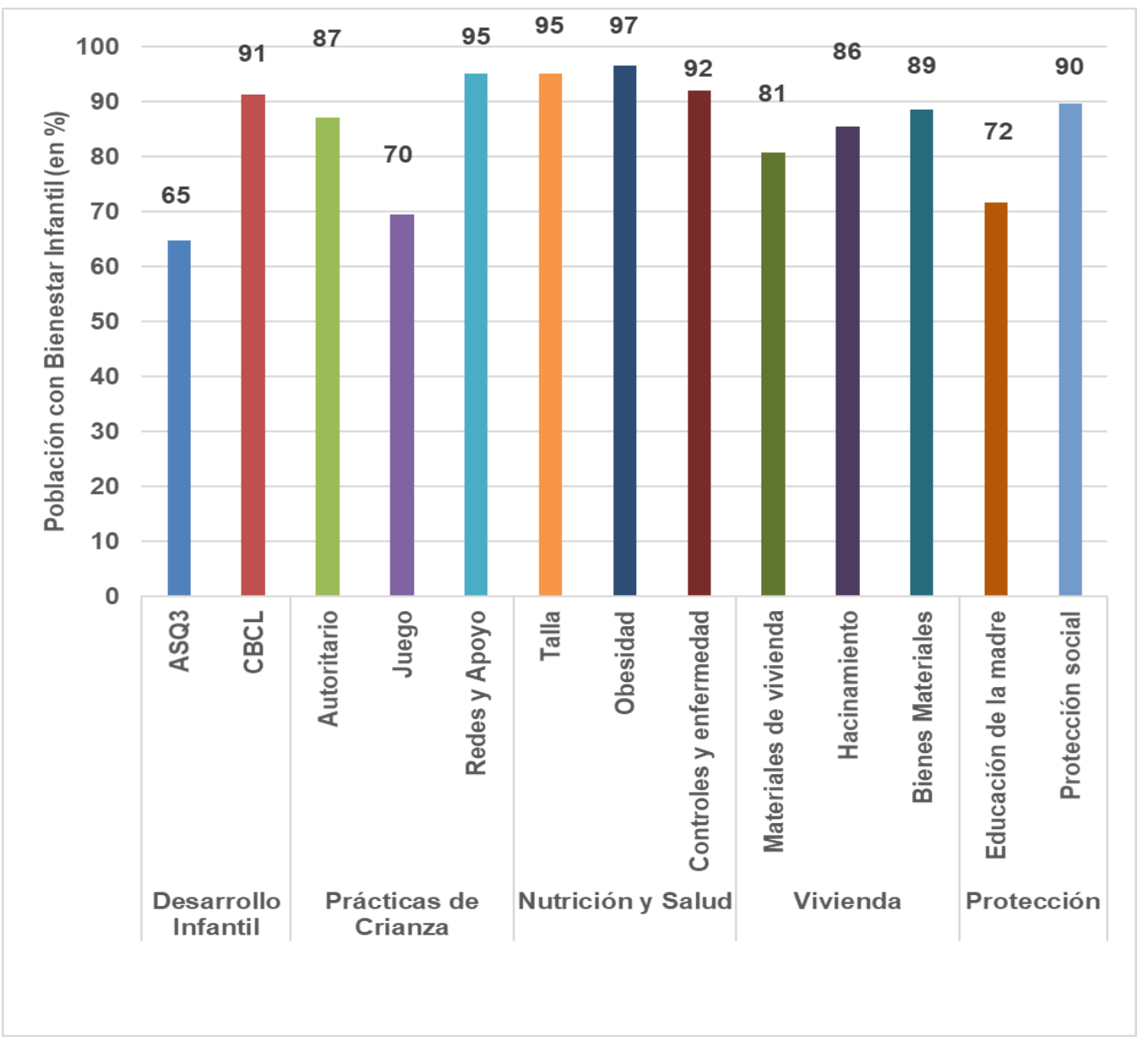

\section{Gráfico 1- Tasas de Incidencia No Censuradas de Bienestar}

Fuente: elaboración propia en base a ENDIS.

\begin{abstract}
11 En el procedimiento de elección de los indicadores y umbrales se descartaron algunos de ellos debido a que contaban con insuficiente número de casos y sus tasas de incidencia eran muy reducidas, sus definiciones no resultaban operativas o eran redundantes en la estimación del índice. De esta forma y a modo de ejemplo, la identificación de si el niño contaba con anemia fue descartado por un número insuficiente de casos, en los indicadores sobre uso del tiempo en tareas de cuidado y apoyo al niño/a que era posible construir en base a la ENDIS no era posible identificar un umbral apropiado y objetivo de acuerdo a la literatura especializada y se consideró la inclusión del indicador ASQ-SE pero el aporte de la información que este brindaba era similar al ASQ3.
\end{abstract}




\section{2. Índice de Bienestar Infantil (IBI)}

En el Índice de Bienestar Infantil (IBI) se utiliza un k=20\%, ${ }^{12}$ lo cual se asocia a que un niño/a cuenta con bienestar infantil si tiene 4 dimensiones sin privaciones, o lo que es equivalente que es carente en bienestar multidimensional si está privado en al menos una dimensión. De acuerdo a los resultados, el IBI asciende en los niños de entre 2 a 6 años de edad a 0.892 . Esto implica que alrededor de un $65.9 \%$ de los niños/as en Uruguay poseen un nivel de bienestar aceptable. Los niños/as con bienestar tienen en promedio un $68.4 \%$ de niveles suficientes en los 11 indicadores considerados (véase Cuadro 4). En oposición, casi 3 de cada 10 niños/as no cuentan con niveles suficientes de bienestar y son considerados como multidimensionalmente pobres. Estos resultados van en línea con los antecedentes para Uruguay. En Colacce y Tenenbaum (2018) se obtiene que 25\% de los niños/as están en situación de pobreza multidimensional en la primera ola de ENDIS. En Failache et al. (2016), que utilizan otra fuente de datos encuentran un porcentaje de niños en situación de pobreza multidimensional más alto de alrededor de $46 \%$.

Cuadro 2. Incidencia e Intensidad en la Población Infantil con y sin Bienestar

\begin{tabular}{clcc}
\hline $\begin{array}{c}\text { Umbral Pobreza } \\
(\mathbf{k})\end{array}$ & \multicolumn{1}{c}{ Índice } & $\begin{array}{c}\text { Con } \\
\text { Bienestar } \\
\text { Infantil }\end{array}$ & $\begin{array}{c}\text { Sin } \\
\text { Bienestar } \\
\text { Infantil }\end{array}$ \\
\hline & Tasa de Incidencia (H, \%) & 65.9 & 34.1 \\
& Intensidad (A, \%) & 68.4 & 31.6 \\
& Índice de Bienestar & 0.892 & 0.108 \\
\cline { 2 - 4 } Observaciones & & 1,499 & 784 \\
\hline
\end{tabular}

Fuente: elaboración propia en base a ENDIS.

A través del Gráfico 2 se observa la proporción de niños/as que alcanza un nivel de bienestar multidimensional según el IBI y tiene suficiencia en cada uno de los indicadores.

\footnotetext{
${ }^{12}$ La selección de este nivel del umbral se fundamenta en que los resultados respecto a la proporción de niños con insuficiencia en el IBI son similares respecto a otros estudios para Uruguay y a que son robustos ante cambios en los ponderadores y según diferentes grupos de población. Se evaluó la posibilidad de construir 3 niveles de bienestar en base a dos puntos de corte (por ejemplo, adicionando un $\mathrm{k}=40 \%$ ), pero para este umbral el número de observaciones era insuficiente para realizar las desagregaciones.
} 
Nuevamente, la dimensión de Nutrición y salud junto con el indicador de Redes y Apoyo son los que presentan tasas de incidencia censuradas más elevadas. En efecto, más de un 95\% de los niños/as alcanzan un nivel de suficiencia en estos indicadores y además tienen bienestar multidimensional en el IBI. En oposición, los indicadores de ASQ3, la educación de la madre y el juego son los que muestran tasas de incidencia censuradas más bajas (del orden de menos de 83\%). Lo que significa que las mayores carencias en los niños de 2 a 6 años provienen de estos indicadores. En consonancia con este resultado, Tuñon et al. (2015) para un indicador similar de estimulación temprana en Argentina donde se incluye también un indicador de juego, plantean que el $18 \%$ de los niños entre 0 a 4 años presentan algún grado de privación en esta dimensión.

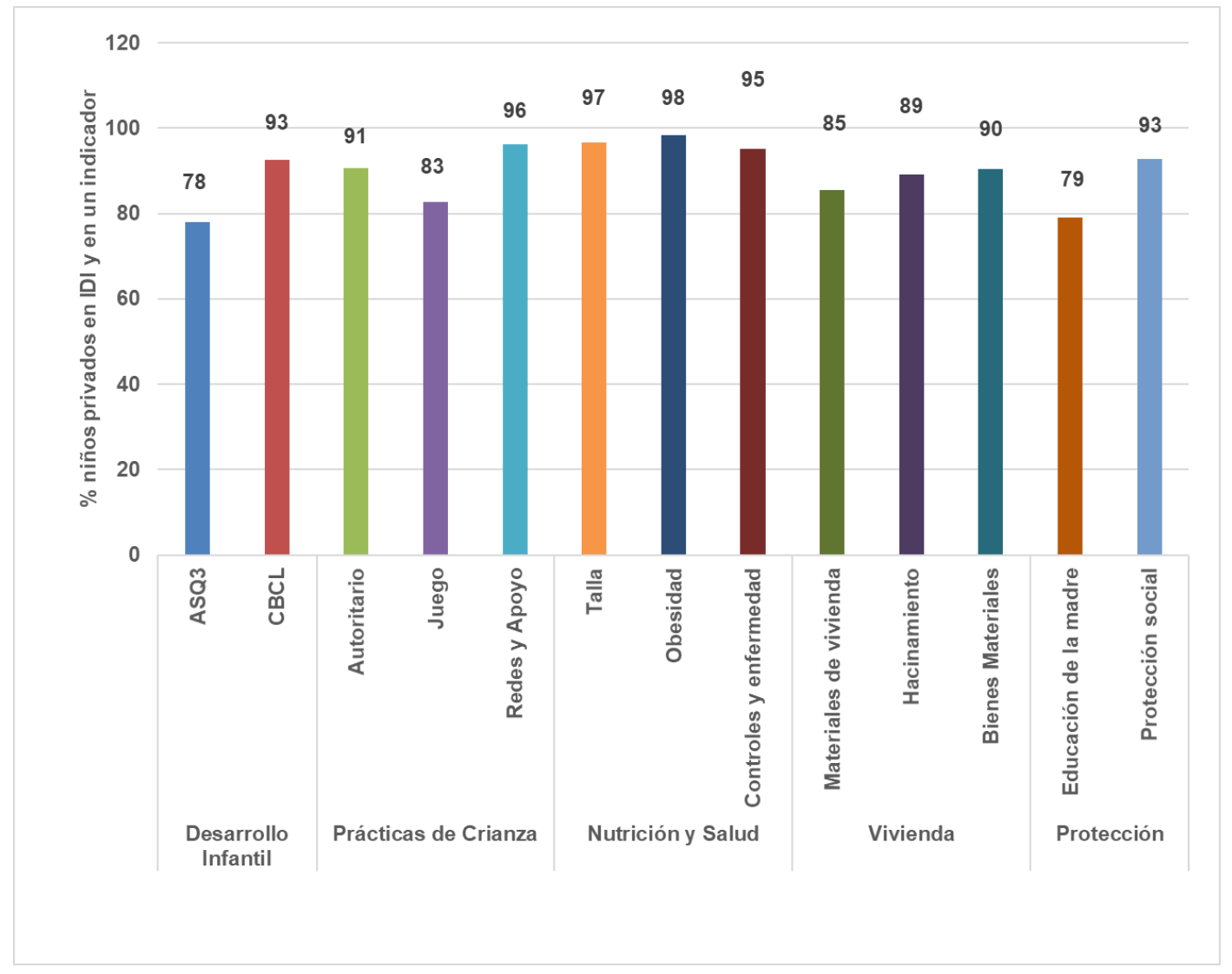

Gráfico 2 - Tasas de Pobreza Censuradas del Índice de Bienestar Infantil (IBI)

Fuente: elaboración propia en base a ENDIS.

La contribución de cada indicador al IBI es relativamente balanceada (Gráfica 3). A su vez, el indicador de ASQ3 y CBCL son los que presentan mayor contribución la que sumada es alrededor de un $21 \%$, participando cada uno en $10.4 \%$, le siguen los indicadores 
de protección social y educación de la madre con cifras cercanas al 9\%. En el caso del resto de los indicadores lo hacen en torno a un $6.2 \%$ y $7.3 \%$.

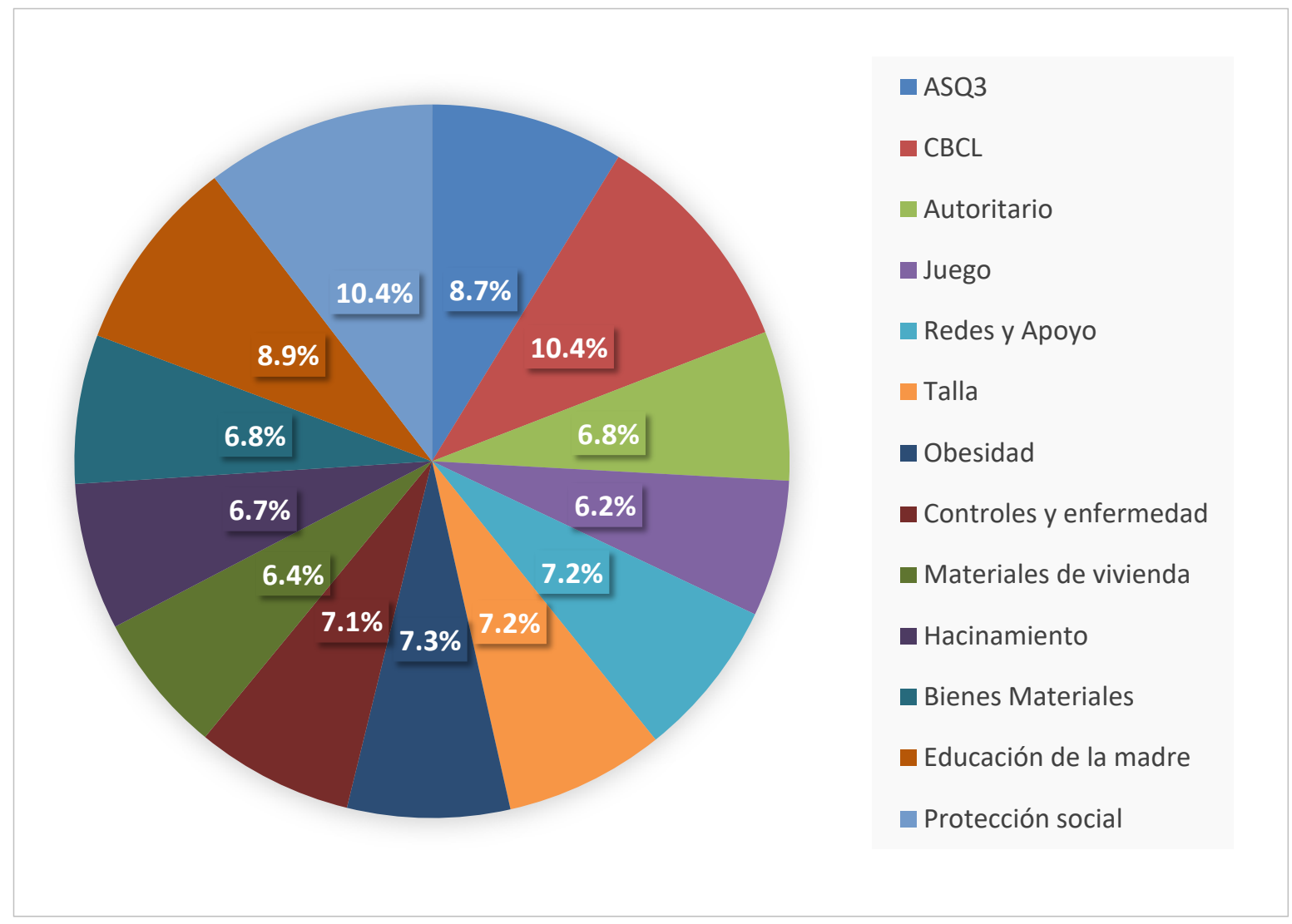

Gráfico 3- Contribución de cada Indicador al Índice de Bienestar Infantil (IBI)

Fuente: elaboración propia en base a ENDIS.

\subsubsection{Intensidad de las privaciones en la población infantil}

Mientras que los niños/as que cuentan con bienestar infantil tienen entre 1 y 2 privaciones como máximo, los que son multidimensionalmente pobres tienen entre 2 y 8 privaciones. En la población con bienestar infantil la mayoría de los niños en este segmento de la población no cuentan con ninguna privación (alrededor de $38 \%$ del total) y es más frecuente que cuenten con una privación que con 2 privaciones (34.8\% vs $27.6 \%$, respectivamente). Por otro lado, la población sin estándares adecuados de bienestar infantil (identificada como multidimensionalmente pobre), muestra una realidad sustancialmente diferente. En efecto, casi la mitad de los niños/as sin bienestar (38.7\%) tienen en promedio 3 privaciones y una proporción pequeña de la población infantil (3\%) está en la situación más crítica (7 y 8 privaciones). 


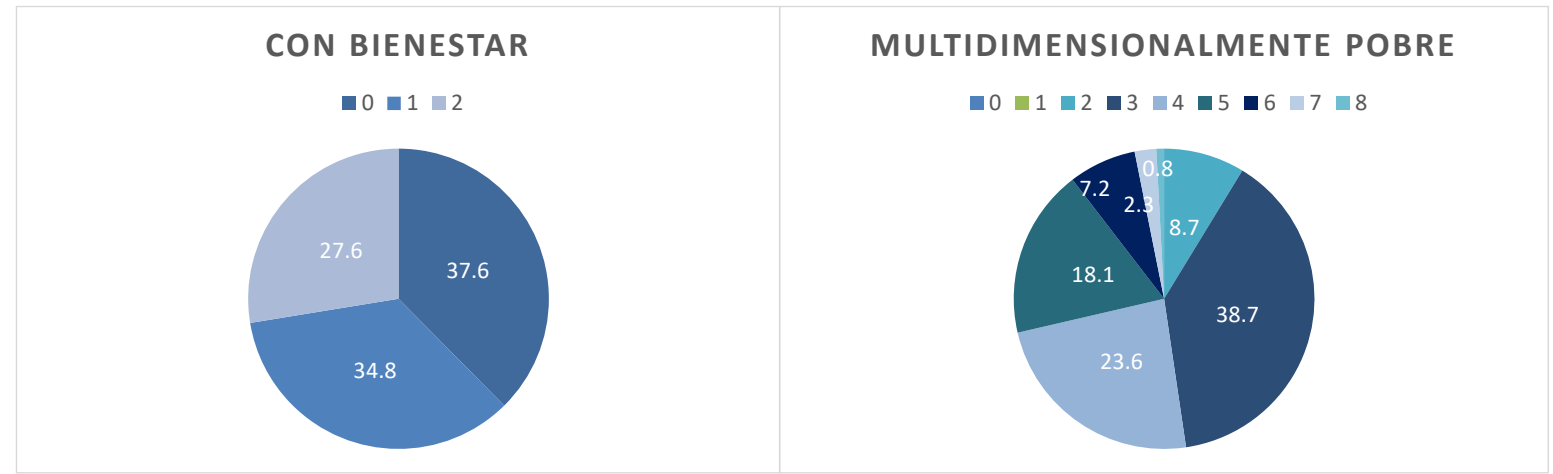

Gráfico 4- Intensidad de privaciones en Niños/as con y sin Bienestar Infantil Fuente: elaboración propia en base a ENDIS.

Al analizar la intensidad de los indicadores, se advierte que los que presentan mayor participación de acuerdo a la diferente cantidad de privaciones son ASQ3, Juego y Educación de la Madre. En los niños/as que no alcanzan un nivel de bienestar suficiente que en promedio cuentan con 7 u 8 privaciones, los indicadores presentan una proporción similar en todos los casos, teniendo participación nula el indicador de obesidad y de controles de enfermedades. Esto reflejaría que para mejorar la condición de carencia de bienestar de la población infantil en situación más crítica sería insuficiente enfocarse en un conjunto reducido de indicadores, sino que por el contrario sería necesario incidir en diversos indicadores de interés.

\subsection{La heterogeneidad de los determinantes del bienestar infantil}

Una de las ventajas de analizar el bienestar a través de la metodología propuesta es que permite realizar desagregaciones además de según dimensiones e indicadores, a través de subgrupos poblacionales ya que el bienestar infantil no ha de manifestarse de igual manera en todos los subgrupos. Se analizan diferentes variables individuales que caracterizan a la población infantil, como sexo, grupo etario, región geográfica y otras características, como si asiste a centros educación inicial en los primeros años de vida, o el bienestar psicológico de la madre.

El bienestar infantil suficiente se presenta en mayor proporción relativa en las niñas, en los grupos etarios de entre 4 y 6 años de edad, en quienes residen en Montevideo, asisten a un centro educativo y si la madre cuenta con bienestar psicológico (Gráfico 5). 


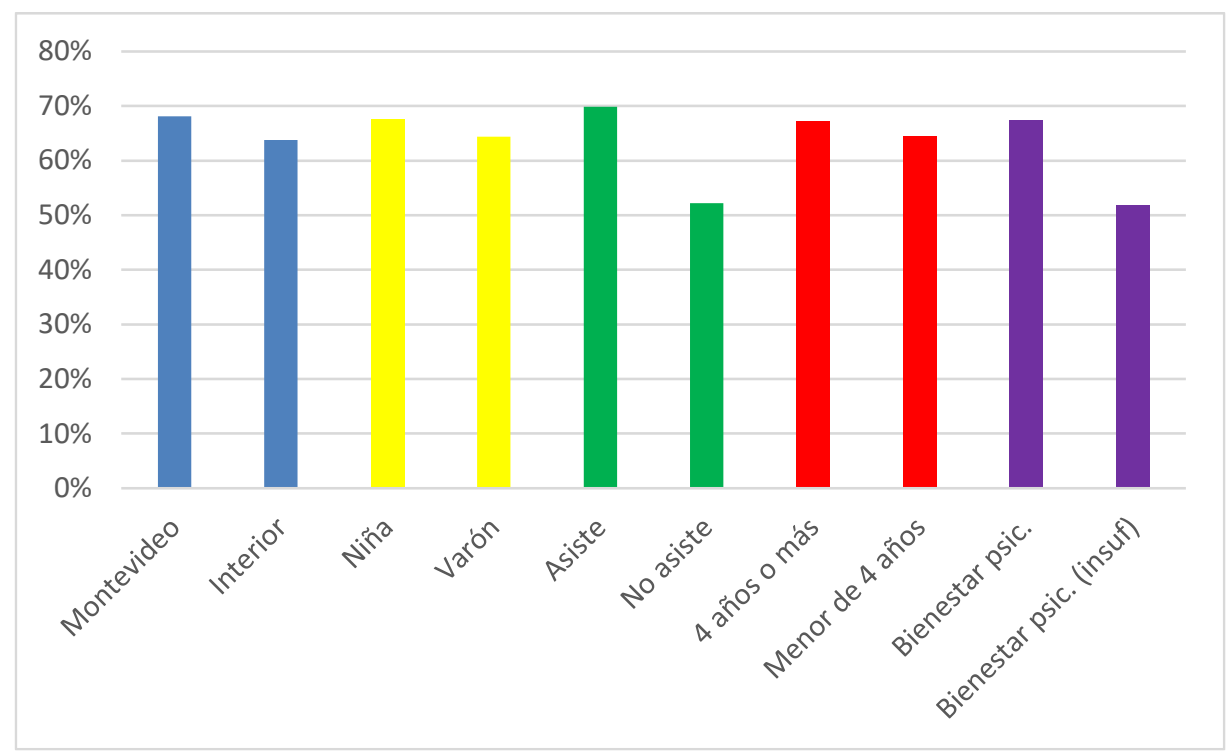

Gráfico 5 - Tasa de Incidencia al bienestar según región, sexo, asistencia, edad y bienestar psicológico de la madre

Fuente: elaboración propia en base a ENDIS.

Si bien los determinantes del bienestar infantil son numerosos y han sido ampliamente estudiados en la literatura, es frecuente que su vínculo sea de interrelación lo cual dificulta la identificación de relaciones de causalidad. Es por este motivo, que el objetivo es poder visualizar la posible asociación que existe entre algunos de estos determinantes. Por un lado, se encuentran los indicadores que integran el IBI y por otro, variables claves que se relacionan con el mismo y que permiten evidenciar la presencia de heterogeneidad en los resultados del índice.

Se utilizan modelos de regresión no lineal Probit para los que la variable dependiente toma el valor de 1 si el niño cuenta con Bienestar Infantil y 0 si es insuficiente considerando que el adulto encuestado es la madre del niño (representando un 96\% de la muestra). En la medida que el IBI se construye en base a diferentes dimensiones, en el modelo de regresión se consideran únicamente algunos de los canales que se asocian con el IBI. Así, se incluyen variables dummies que remiten tanto a características individuales del niño/a (sexo, grupos de edad, región y tipo de centro educativo al que asiste), al vínculo con el padre del niño (si ve al padre con baja frecuencia y la relación con el padre ${ }^{13}$ ) y sobre el entorno (educación de

${ }^{13}$ El niño/a ve al padre con baja frecuencia $(=1)$ si lo ve menos de 2 días a la semana o no lo ve. La relación tensa con el padre $(=1)$ implica que con frecuencia o siempre están en desacuerdo en temas básicos o la conversación es tensa u hostil. 
la abuela del niño/a y si el hogar donde creció la madre era conflictivo ${ }^{14}$ ) y rasgos psicológicos y de personalidad de la madre. En el Cuadro 3 se presentan los resultados de la estimación de un modelo de regresión bajo cinco especificaciones diferentes.

Al contemplar una especificación básica donde solo se consideren variables relativas a características del niño/a (columna 1), únicamente si el centro educativo al que concurre es público arroja un resultado estadísticamente significativo el cual se asocia a menor bienestar relativo respecto a si concurre a un centro privado. Salas (2016) analiza el rol de la educación temprana en el desarrollo de las habilidades cognitivas y no cognitivas de los niños de 2 y 3 años, encontrando que la asistencia educativa en edades tempranas puede moldear el comportamiento de los niños dentro del hogar, reforzando el estímulo que reciben en los hogares y en algunos casos compensando el mismo. En la columna 1 del Cuadro 3 se presentan los resultados de incorporar además las características del vínculo con el padre, obteniendo que el hecho de tener una relación tensa con este o ver al padre con baja frecuencia reduce el bienestar.

Al considerar las características del entorno del adulto encuestado se obtiene que si el hogar donde la madre creció era conflictivo se relaciona con una reducción de la probabilidad de contar con bienestar infantil, a la vez que aumenta conforme se incrementa el máximo nivel educativo de la abuela del niño (columna 2)

Al analizar las características psicológicas y de personalidad, los coeficientes del indicador en base al test de SRQ y Big Five Inventory son estadísticamente significativos separadamente (Especificación 3 y 4). No obstante, al incluir ambos (columna 5), la variable de bienestar psicológico pierde significatividad y son el Neuroticismo y la Apertura estadísticamente significativos, siendo la asociación con el bienestar infantil del primero negativa y del segundo positiva. Estos resultados son razonables dada la correlación existente entre el indicador de bienestar psicológico y el de neuroticismo del BFI, lo que es ya mencionado en Perazzo et al. (2019). Los autores a su vez obtienen resultados que van en sintonía con los encontrados, mediante una asociación entre el desarrollo infantil con el test CBCL y el componente de neuroticismo del BFI y el SRQ se relacionan de forma positiva con los problemas externalizados e internalizados del niño, y el componente de apertura del $\mathrm{BFI}$ asociado a menores problemas del CBCL.

\footnotetext{
${ }^{14}$ Se incluye máximo nivel educativo de la abuela materna del niño/a siendo la variable omitida si no tiene instrucción. La variable conflicto releva la opinión de la madre respecto a que el hogar en donde creció existía mucho conflicto $(=1)$, en relación a poco o ningún tipo de conflicto $(=0)$.
} 
Cuadro 3. Estimaciones de modelos de regresión de variables relacionadas al IBI

\begin{tabular}{|c|c|c|c|c|c|}
\hline & (1) & $(2)$ & (3) & $(4)$ & (5) \\
\hline $\begin{array}{l}1 \text { - Características individuales } \\
\text { Centro educativo público } \\
\text { (ANEP; municipal o CAIF) }\end{array}$ & $\begin{array}{l}-0.28^{* * * *} \\
(0.026) \\
\end{array}$ & $\begin{array}{l}-0.22 * * * \\
(0.028) \\
\end{array}$ & $\begin{array}{l}-0.22 * * * \\
(0.028) \\
\end{array}$ & $\begin{array}{l}-0.21 * * * \\
(0.027) \\
\end{array}$ & $\begin{array}{r}-0.21 * * * \\
(0.027) \\
\end{array}$ \\
\hline \multicolumn{6}{|c|}{2 - Características del vínculo con el padre (pareja) del niño/a } \\
\hline $\begin{array}{l}\text { Ve al Padre con Baja } \\
\text { Frecuencia }\end{array}$ & $\begin{array}{c}-0.16^{* * * *} \\
(0.031)\end{array}$ & $\begin{array}{l}-0.15^{* * *} \\
(0.031)\end{array}$ & $\begin{array}{l}-0.15^{* * * *} \\
(0.031)\end{array}$ & $\begin{array}{l}-0.14 * * * \\
(0.032)\end{array}$ & $\begin{array}{l}-0.14 * * * \\
(0.032)\end{array}$ \\
\hline $\begin{array}{l}\text { Desacuerdo o relación } \\
\text { tensa con la pareja }\end{array}$ & $\begin{array}{l}-0.11 * * * \\
(0.038) \\
\end{array}$ & $\begin{array}{l}-0.11 * * * \\
(0.039) \\
\end{array}$ & $\begin{array}{l}-0.11 * * * \\
(0.039) \\
\end{array}$ & $\begin{array}{l}-0.09 * * \\
(0.039) \\
\end{array}$ & $\begin{array}{l}-0.09 * * \\
(0.039) \\
\end{array}$ \\
\hline \multicolumn{6}{|c|}{3 - Entorno y características del adulto encuestado } \\
\hline \multicolumn{6}{|c|}{ Máximo Nivel Educativo de la Madre (del encuestado) } \\
\hline Primaria & & $\begin{array}{c}-0.02 \\
(0.100)\end{array}$ & $\begin{array}{l}-0.00 \\
(0.102)\end{array}$ & $\begin{array}{l}-0.04 \\
(0.099)\end{array}$ & $\begin{array}{c}-0.03 \\
(0.101)\end{array}$ \\
\hline Secundaria Incompleta & & $\begin{array}{c}0.09 \\
(0.103)\end{array}$ & $\begin{array}{c}0.10 \\
(0.104)\end{array}$ & $\begin{array}{c}0.06 \\
(0.102)\end{array}$ & $\begin{array}{c}0.07 \\
(0.103)\end{array}$ \\
\hline Secundaria Completa & & $\begin{array}{c}0.15 \\
(0.105)\end{array}$ & $\begin{array}{c}0.17 \\
(0.106)\end{array}$ & $\begin{array}{c}0.11 \\
(0.105)\end{array}$ & $\begin{array}{c}0.11 \\
(0.106)\end{array}$ \\
\hline Terciaria & & $\begin{array}{c}0.16 \\
(0.104)\end{array}$ & $\begin{array}{c}0.17 \\
(0.106)\end{array}$ & $\begin{array}{c}0.13 \\
(0.103)\end{array}$ & $\begin{array}{c}0.14 \\
(0.104)\end{array}$ \\
\hline Superior Completa & & $\begin{array}{l}0.26^{* * *} \\
(0.101)\end{array}$ & $\begin{array}{l}0.28 * * * \\
(0.103)\end{array}$ & $\begin{array}{l}0.22^{* *} \\
(0.102)\end{array}$ & $\begin{array}{l}0.23 * * \\
(0.103)\end{array}$ \\
\hline $\begin{array}{l}\text { Era conflictivo el hogar } \\
\text { donde creció }\end{array}$ & & $\begin{array}{r}-0.14 * * * \\
(0.033)\end{array}$ & $\begin{array}{l}-0.13 * * * \\
(0.034)\end{array}$ & $\begin{array}{l}-0.12 * * * \\
(0.034)\end{array}$ & $\begin{array}{l}-0.12 * * * \\
(0.034)\end{array}$ \\
\hline \multicolumn{6}{|c|}{ 3.2 Características psicológicas y de personalidad } \\
\hline \multicolumn{6}{|l|}{ Bienestar psicológico } \\
\hline $\mathrm{SRQ}$ & & & $\begin{array}{l}-0.10^{* *} \\
(0.039)\end{array}$ & & $\begin{array}{c}-0.06 \\
(0.039)\end{array}$ \\
\hline \multicolumn{6}{|l|}{ Big Five Inventory } \\
\hline Extraversion & & & & $\begin{array}{c}-0.00 \\
(0.002)\end{array}$ & $\begin{array}{c}-0.00 \\
(0.002)\end{array}$ \\
\hline Amabilidad & & & & $\begin{array}{c}-0.00 \\
(0.003)\end{array}$ & $\begin{array}{c}-0.00 \\
(0.003)\end{array}$ \\
\hline Responsabilidad & & & & $\begin{array}{c}0.00 \\
(0.003)\end{array}$ & $\begin{array}{c}0.00 \\
(0.003)\end{array}$ \\
\hline Neuroticismo & & & & $\begin{array}{c}-0.01 * * * \\
(0.002)\end{array}$ & $\begin{array}{c}-0.01 * * * \\
(0.002)\end{array}$ \\
\hline Apertura & & & & $\begin{array}{c}0.01^{* * *} \\
(0.002)\end{array}$ & $\begin{array}{c}0.01^{* * * *} \\
(0.002) \\
\end{array}$ \\
\hline Observaciones & 1,812 & 1,609 & 1,609 & 1,609 & 1,609 \\
\hline R-squared & 0.108 & 0.168 & 0.171 & 0.197 & 0.198 \\
\hline
\end{tabular}

Errores estándar robustos entre paréntesis

${ }^{* * *} p<0.01,{ }^{* *} p<0.05,{ }^{*} p<0.1$

Fuente: elaboración propia en base a ENDIS. 


\section{Comentarios Finales}

En países en desarrollo el bienestar en la infancia continúa siendo un tema relevante y por lo tanto comprender de forma exhaustiva las dimensiones que la definen en términos multidimensionales es pertinente. Los estudios vinculados al bienestar infantil se han incrementado en el último tiempo, en parte debido a la mayor disponibilidad y riqueza de información. Este es el caso de la Encuesta de Nutrición, Desarrollo Infantil y Salud (ENDIS) de Uruguay que permite realizar un estudio comprehensivo de la primera infancia en múltiples dimensiones del bienestar. Es así que el objetivo de este estudio es plantear cuáles son las dimensiones que mejor se ajustan y reflejan el bienestar multidimensional en la primera infancia mediante un Índice de Bienestar Infantil (IBI) utilizando la metodología de Alkire y Foster (2011), permitiendo caracterizar de forma lo más abarcativa posible la situación de la primera infancia. A la vez, se buscó conocer algunos de los canales por los cuales se ven afectadas dichas dimensiones y las asociaciones condicionadas a un conjunto de variables en el bienestar de la primera infancia y la presencia de ciertos grupos que presentan rasgos comunes que podrá ser de utilidad en el diseño de políticas públicas mejor orientadas. En este estudio y en general en los estudios de esta naturaleza la selección de dimensiones e indicadores es compleja dado que aun incluyendo las dimensiones más frecuentes es debatible si las mismas son parte constitutiva del bienestar infantil o están asociadas al mismo. Esta es una limitación importante del presente estudio que intentamos abordar mediante una amplia discusión sobre las dimensiones seleccionadas y los canales asociados.

Alrededor de un $66 \%$ de los niños/as en Uruguay poseen un nivel de bienestar aceptable, definido el umbral como aquellos que presentan 4 dimensiones sin privaciones, o lo que es equivalente que son carentes en bienestar multidimensional si se encuentran privados en al menos una dimensión. Los indicadores vinculados al desarrollo infantil son los que más contribuyen, a su vez se encuentra una mayor incidencia del bienestar en las niñas, en edades más grandes, en aquellos que asisten a un centro educativo y en madres con bienestar psicológico.

Intervenciones focalizadas en pocas dimensiones del bienestar podrían redundar en mejoras relativamente efectivas. Si bien se considera necesario continuar profundizando en cada una de estas dimensiones y sus relaciones, el trabajo se plantea como un avance para poder repensar acciones y políticas entorno a los primeros años de vida de los niños como pueden ser las políticas de cuidados (licencias parentales, centros de estimulación oportuna, asistencia educativa temprana). La disponibilidad de la tercera ola de información de esta misma cohorte permitirá continuar el análisis de estos niños y niñas en etapa escolar, y de ese modo poder estudiar si las dimensiones relevantes del bienestar se ven modificadas, así 
como si las privaciones en los primeros años de vida continúan teniendo efectos en el bienestar o algunas de ellas se diluyen en el tiempo.

\section{Referencias bibliográficas}

Ahnert, L. y Lamb, M. (2003). Shared care: Establishing a balance between home and child care settings. Child Development, 74, 1044-1049.

Almond, D., y Currie, J. (2011). Killing Me Softly: The Fetal Origins Hypothesis. Journal of Economic Perspectives, American Economic Association, 25(3),153-172.

Alkire, S. y Foster, J. (2011). Counting and Multidimensional Poverty Measurement. Journal of Public Economics, 95(7-8), 476-487.

Alkire, S. y Roche, J. M. (2011). Beyond headcount: measures that reflect the breadth and components of child poverty. OPHI Working Paper 45.

Alkire, S., Lham, D., Gyeltshen, S. y Minten, T. (2016). Child Poverty in Buthan: Insights from Multidimensional Child Poverty Index (C-MPI) and Qualitative Interviews with poor children. National Statistics Bureau.

Alves, G. y Zerpa, M. (2011). Pobreza en la adolescencia en áreas rurales y urbanas en Uruguay. IECON Serie Documentos de Trabajo No. 4/11.

Andresen S., Diehm I., Sander, U. y Ziegler, H. (2010). Children and the Good Life: New Challenges for Research on Children. Dordrecht: Springer Netherlands.

Apablaza, M. y Yalonetzky, G. (2011). Measuring the dynamics of multiple deprivations among children: the cases of Andhra Pradesh, Ethiopia, Peru and Vietnam. Young Lives Research in Progress, Oxford: University of Oxford.

Arranz Freijo, E. (2004). Un modelo teórico para la comprensión de las relaciones entre la interacción familiar y el proceso de desarrollo psicológico modelo contextualecológico, interactivo-bidireccional y sistémico. En E. Arranz Freijo (Coord.), Familia y desarrollo psicológico (32-69). Madrid: Pearson educación.

Berlinski, S. y Schady, N. (2015). Los primeros años: El bienestar infantil y el papel de las políticas públicas. Banco Interamericano de Desarrollo, BID.

Biggeri, M. (2004). The capability approach and children well-being. Dipartimento di Scienze Economiche, Studi e discussioni No. 141.

Biggeri, M, Ballet, J y Comim, F. (2011). The Capability Approach and Research on Children: Capability Approach and Children's Issues. En S. Andresen, I. Diehm, 
U. Sander y H. Ziegler (Eds.), Children and the Good Life: New Challenges for Research on Children (pp. 75-89). Vol. 4. Springer.

Blau, D. y Currie, J. (2006). Who is minding the kids. En E. Hanushek y F. Welch (Eds.), The Handbook of the Economics of Education (1163-1278). New York: North Holland.

Castillo, J. y Colombo, K. (2014). Pobreza en niños en Uruguay: Caracterización y determinantes desde una perspectiva dinámica. Tesis para la obtención del título de Licenciado en Economía. FCEyA, UdelaR.

Colacce, M. y Tenenbaum, V. (2017). Un análisis de la evolución de la pobreza multidimensional en la infancia y adolescencia en Uruguay entre 2006 y 2014. Estudios Económicos, 32(2), 171-212.

Colacce, M. y Tenenbaum, V. (2018). Las dimensiones del bienestar infantil y la focalización de los programas dirigidos a la primera infancia. Serie Estudios y Perspectivas 36. Santiago de Chile: CEPAL.

Conti, G. y Heckman, J. (2012). The Economics of Child Well-Being. IZA Discussion Papers 6930, Institute for the Study of Labor (IZA).

Cunha, F., Heckman, J. y Lochner, L. y Masterov, V. (2006). Interpreting the Evidence on Life Cycle Skill Formation. En E. Hanushek y F. Welch (Eds.), Handbook of the Economics of Education (697-812). New York: North Holland

Espíndola, E., Sunkel, G., Murden, A. y Milosavljevic, V. (2017). Medición multidimensional de la pobreza infantil. Una revisión de sus principales componentes teóricos, metodológicos y estadísticos. CEPAL y UNICEF.

Failache, E., Salas, G. y Vigorito, A. (2016). La dinámica reciente del bienestar de los niños en Uruguay. Un estudio en base a datos longitudinales. IECON Serie Documentos de Trabajo No. 11/16.

Felfe, C. y Lalive, R. (2014). Does Early Child Care Help or Hurt Children's Development? IZA Discussion Paper No. 8484.

Main, G. y Bradshaw, J. (2012). A child material deprivation index. Child Indicators Research, (5), 503-521. https://doi.org/10.1007/s12187-012-9145-7

Marroig, A., Perazzo, I., Salas, G. y Vigorito, A. (2017). Evaluación de impacto del programa de acompañamiento familiar de Uruguay Crece Contigo. Serie Documentos de Trabajo DT 15/2017. Instituto de Economía, Facultad de Ciencias Económicas y de Administración, Universidad de la República. 
Minujin, A., Delamonica, E., Davidziuk, A. y Gonzalez, D. (2006). The definition of child poverty: a discussion of concepts and measurements. Environment and Urbanization, 18(2), 481-500.

Nathan, M. y Zerpa, M. (2011). Pobreza en la infancia y la adolescencia en Montevideo y el área metropolitana. Un análisis multidimensional. En Grupo Interdisciplinario de Estudios de Familia (edit.), Cambio familiar y bienestar de las mujeres y los niños en Montevideo y el área metropolitana. Una perspectiva longitudinal. UdeLaRUNICEF (en prensa).

Nussbaum, M. (2000). Women and human development: The capabilities approach. Cambridge: Cambridge University Press.

Perazzo, I., Salas, G. y Sena, E. (2019). Dynamics of the parenting practices and child development. DT 21/19. Instituto de Economía. Facultad de Ciencias Económicas y de Administración, Universidad de la República.

Pérez, T. (2016). Determinants of child poverty in Uruguay. The impact of gender inequality. Tesis de Maestría in Economic, Development and Growth, School of Economics and Management, Lund University.

Robeyns, I. (2003). The Capability Approach: An Interdisciplinary Introduction. Amsterdam: University of Amsterdam.

Roelen, K., Gassmann, F. y de Neubourg. C. (2012). False positives or hidden dimensions: What can monetary and multidimensional measurement tell us about child poverty in Vietnam? International Journal of Social Welfare, 21, 393-407.

Salas, G. (2016). Early childhood development, school attendance and parenting. Capítulo 2, Tesis para la obtención del título de Doctorado en Economía, Universidad Autónoma de Barcelona.

Sen, A. K. (1992). Inequality Re-examined. Oxford: Clarendon Press.

Sen, A. K. (1993). Capability and well-being. En M. Nussbaum y A. K. Sen (Eds.), The Quality of Life (pp. 30-53). Oxford: Clarendon Press.

Stefánsson, K. L., Arnardóttir y Karlsson, A. (2018). Children‘s Deprivation and Economic Vulnerability in Iceland 2009 and 2014. Child Indicators Research, 11(3), 783803.

Torres, L. (2014). Pobreza multidimensional de la infancia en Colombia. Tesis doctoral, Universidad de La Sabana. 
Trani, J. y Cannings T. (2013). Child Poverty in an Emergency and Conflict Context: A Multidimensional Profile and an Identification of the Poorest Children in Western Darfur. World Development, 48(C), 48-70.

Tuñón, I., Poy, S. y Coll, A. (2015). Pobreza infantil en las ciudades de la Argentina 20102014: Diferentes mediciones de la pobreza infantil y una propuesta multidimensional desde un enfoque de derechos. Edición especial - Ciudad Autónoma de Buenos Aires: Educa.

Ura, K., Alkire, S. y Zangmo, T. (2012). GNH and GNH Index. The Centre for Buthan Studies.

Urzua, S. y Veramendi, G. (2011). The Impact of Out-of-Home Childcare Centers on Early Childhood Development. IDB Working Paper Series, No. IDB-WP-240.

\section{Otros documentos consultados}

CEPAL y UNICEF (2010). Pobreza infantil en América Latina y el Caribe. Santiago: Naciones Unidas.

Grupo de Estudios de Familia (2015), Salud, Nutrición y Desarrollo en la Primera Infancia en Uruguay, Primeros Resultados de la ENDIS.

UNICEF (2012). Child Development and Economic Development: Lessons and Future Challenges. Child Poverty Insights. Jo Boyden and Stefan Dercon.

WHO (1994). A User's Guide to the self reporting questionnaire. Division of Mental Health, World Health Organization, Genova. 